\title{
Sexual dimorphism in LEC rat liver: Suppression of carbonic anhydrase III by copper accumulation during hepatocarcinogenesis
}

\author{
Makihiko Kuhara ${ }^{1,3}$, Jingshu Wang ${ }^{2}$, Maria Jolina Flores ${ }^{2}$, Zhiwei Qiao ${ }^{2}$, Yukio Koizumi ${ }^{2}$, Souichi Koyota ${ }^{2}$, \\ Naoyuki Taniguchi ${ }^{1}$, and Toshihiro SugiYama ${ }^{1,2}$ \\ ${ }^{1}$ Department of Biochemistry, Osaka University Graduate School of Medicine, 2-2 Yamadaoka, Suita, Osaka 565-0871, Japan; ${ }^{2}$ Depart- \\ ment of Biochemistry, Akita University Graduate School of Medicine, 1-1-1 Hondo, Akita 010-8543, Japan; and ${ }^{3}$ Department of Clini- \\ cal Nutrition, Osaka Prefecture University, 3-7-30 Habikino, Osaka 538-8555, Japan
}

(Received 2 December 2010; and accepted 27 December 2010)

\begin{abstract}
We examined age-related changes in the protein expression of carbonic anhydrase III (CAIII) in livers of Long-Evans with a cinnamon-like color (LEC) rats using an agouti color (LEA) rats as controls. The levels of the protein of CAIII in the liver of LEC male rats increased before 20 weeks of age, at the stage of acute hepatitis, and were decreased at 54 weeks of age, while those of CAIII in the liver of LEA male rats were highly expressed at all ages. In the normal LEA rats, CAIII showed sexual dimorphism. The level of CAIII in LEA male rat liver relative to female was four times higher. On the other hand, young LEC rat (at 4-12 weeks) showed a higher protein level of CAIII than LEA rats, and then decreased during development of hepatitis. CAIII mRNA also decreased in the LEC rat liver during hepatocarcinogenesis. The level of CAIII in the tumor region was lower than that in the tumor-free region. Immunohistochemical analysis showed that glutathione $S$-transferase P (GST-P) was positive and CAIII was negative in the precancerous region. The expression of CAIII was suppressed in cancerous lesions in hepatoma-bearing LEC rat liver compared to uninvolved surrounding tissues. These results indicated that suppression of CAIII accompanied hepatocarcinogenesis and it is a secondary consequence of the high copper levels in the liver.
\end{abstract}

Carbonic anhydrase (CA) [EC 4, 2, 1, 1] is a widely distributed enzyme in animals and plants. Numerous isoforms of CA have been identified and determined at both the protein level and the DNA level $(3,5$, $14,16)$. The expression patterns of CA are different in each tissue. CAI and CAII are predominant in erythrocytes $(2,8)$, while CAIII is expressed in the liver and muscle $(12,22,24)$. Rat liver CAIII exhibits sexual dimorphism. Jeffery et al. (12) have shown that CAIIl concentrations in males are correlated with the production of testosterone and pattern

Address correspondence to: Dr. Toshihiro Sugiyama, Department of Biochemistry, Akita University Graduate School of Medicine, 1-1-1 Hondo, Akita 010-8543, Japan Tel: +81-18-884-6074, Fax: +81-18-884-6443

E-mail: sugiyama@med.akita-u.ac.jp of growth hormone release. No sexual dimorphism at 21 days was found, but from 50 to 400 days CAIII shows sexual difference. CAIII occurs in male rat liver at concentrations twenty times those in the female. Their group (11) has also shown that CAIIl expression is highly sensitive to the endogenous growth hormone secretory pattern. Males release growth hormone episodically and have high concentrations of CAIII; however females produce growth hormone in a more continuous fashion and have lower CAIII levels.

CAIII is a major cytosolic protein accounting for approximately $8 \%$ of the protein in rat liver cytosol (28). The carbonic anhydrases catalyze the reversible reaction of carbon dioxide and water to form carbonic acid. In addition, CAIII was reported to play a role of an antioxidant that prevents $\mathrm{H}_{2} \mathrm{O}_{2}$ - 
inducible apoptosis and a coplanar polychlorinated biphenyl, 3, 3', 4, 4', 5-pentachlorobiphenyl (PenCB) treatment on the rats results in reduced glutathione peroxidase-redox system and catalase. PenCB significantly suppresses the expression of rat liver CAIII (8). Oxidative stress is one of the toxicities caused by many polycyclic compouds.

LEC (Long-Evans with a cinnamon coat color) rats suffer from hereditary hepatitis and subsequent liver cancer at a high frequency $(19,20,25,37)$. Hepatitis suddenly appears approximately 12-16 weeks after birth and has a high lethality. The remaining animals survive more than one year and all develop hepatocellular carcinoma spontaneously, attributed to abnormal copper metabolism. This mutant strain of rat shows some clinical features that are similar to those of Wilson disease. Molecular studies have revealed that the copper transporting P-type ATPase (Atp7b) was defective in the LEC rat. These observations have confirmed that the LEC rat is a rodent model for Wilson disease. In addition, recent studies have suggested that the human ATP7B protein is involved in the intracellular transport of hepatic copper. The absence or diminution of Atp7B and ATP7B function results in abnormal copper metabolism in the LEC rat and in patients with Wilson disease (ATP7B), respectively. The gene responsible for Wilson disease, located on chromosome 13, was identified in 1993 by positional cloning and linkage disequilibrium analysis $(23,35)$ and by searching for genes with homology to ATP7A, the gene for the X-linked disorder of copper metabolism in Menkes disease.

There have been many reports about sexual dimorphism in LEC rats $(7,13,27)$. We recently showed that the pattern of expressions of cytochrome P450 isoforms can be feminized during development of hepatitis and hepatoma (7). In the present study, we investigated sexual dimorphism of CAIII in LEC rat liver during hepatocarcinogenesis using normal LEA (Long-Evans with an agouti coat color) rat liver as a control, and we found that CAIII was suppressed by copper accumulation during hepatocarcinogenesis.

\section{MATERIALS AND METHODS}

Animals. The LEC rats were maintained under normal conditions at the Institute of Experimental Animal Sciences, Osaka University Graduate School of Medicine and Akita University Graduate School of Medicine. All research procedures complied with the ethical standards set in the Helsinki Declaration of 1975. Detailed information on the origin, maintenance, and breeding history of the LEC rats has been previously described $(25,37)$. Long-Evans Agouti (LEA) rats, which are a sibling line of LEC rats and do not develop hepatitis and hepatocellular carcinoma, were used as a control. The LEC and LEA rats used in this study were at the 50th and 49th generations of brother-sister mating, respectively. We used male and female LEC rats from aged 4 weeks to 16 weeks that had not yet developed acute hepatitis, aged 8 months with chronic hepatitis, and after those ages with hepatocellular carcinoma.

Sample collection. Animals were anesthetized with diethyl ether and the liver was perfused with cold physiological saline $(0.86 \% \mathrm{NaCl})$ and homogenized in five volumes of cold $0.25 \mathrm{M}$ sucrose containing $5 \mathrm{mM}$ Tris-HCl $(\mathrm{pH} \mathrm{7.4)}$ and $1 \mathrm{mM}$ EDTA with a Potter-Elvehjem homogenizer. The homogenates were centrifuged at $9,000 \times g$ for $15 \mathrm{~min}$, and the resulting postmitochondrial supernatant fraction was centrifuged at $105,000 \times g$ in a Hitachi RP-55T rotor for $1 \mathrm{~h}$. The cytosolic fractions were stored at $-80^{\circ} \mathrm{C}$ for up to one month until analysis. Since hyperplastic nodules formed the tumors with a white/ reddish smooth surface, it can be excised visually. The hyperplastic nodules and the surrounding normal tissues were excised carefully under a stereoscopic microscope from the three livers of each male and female LEC rats at 52 weeks.

Western blotting. Sodium dodecyl sulfate polyacarylamide gel electrophoresis (SDS-PAGE) was conducted in $15 \%$ gel according to the method of Laemmli (15). Cells were lysed in cell lysis buffer $[50 \mathrm{mM}$ Tris- $\mathrm{HCl}$ (pH 7.4), 0.1\% SDS, 1\% NP40, $0.5 \%$ deoxycholic acid sodium salt, and $150 \mathrm{mM}$ $\mathrm{NaCl}$. Fifty $\mu \mathrm{g}$ of protein per lane were then separated by SDS-PAGE and blotted onto PVDF membranes, which were subsequently saturated with $10 \%$ skimmed milk in Tris-buffered saline containing $0.5 \%$ Tween 20 . The membrane was then incubated overnight at $4^{\circ} \mathrm{C}$ in a sealed plastic bag with $75 \mathrm{~mL}$ of buffer A (PBS, $0.05 \%$ Tween 20) containing the rabbit antibody to equine CA III purified from equine liver cytosol (22). The membranes were washed four times with buffer A for $10 \mathrm{~min}$ at room temperature and then incubated in a sealed bag with $75 \mathrm{~mL}$ of buffer A containing 1000-fold diluted peroxidase-conjugated goat anti-rabbit IgG (Dako Japan, Kyoto, Japan) for $3 \mathrm{~h}$ at room temperature. The membrane was again washed three times with buffer A for $10 \mathrm{~min}$ at room temperature and once with 
PBS. The membrane was then incubated in $4 \mathrm{mM}$ 4-chioro-1-naphthol and $0.01 \% \mathrm{H}_{2} \mathrm{O}_{2}$ in PBS.

Immunohistochemical staining for CAIII and GST-P. Glutathione $S$-transferase P (GST-P) was observed in the hyperplastic foci and nodules induced by chemical carcinogens $(23,24)$. Hyperplastic nodules were observed in all of the 52-week old LEC rats. Hyperplastic nodules and the surrounding normal tissues were excised carefully under a stereoscopic microscope from the livers of the 52-week old rats. Liver tissues were fixed in $4 \%$ paraformaldehyde. Specimens were embedded in paraffin. Then $5 \mu \mathrm{m}$ sections were cut and deparafinized. Liver sections were treated sequentially with skim milk, rabbit anti-GST-P (BIOPRE, Dublin, Ireland; 1000-fold dilution), biotin-labeled goat anti-rabbit IgG (Dako Japan, Kyoto, Japan; 1,000-fold dilution) and avidinbiotin-peroxidase (LSAB Kit; Dako Japan) was used to demonstrate GST-P positive liver cells. Sections are then counter-stained with hematoxylin for microscopic examination. The cytological images observed with a microscope (BH2; Olympus, Tokyo Japan) via a TV camera (Ikegami ITC-350M, Tokyo Japan).

Assay of testosterone concentration. Testosterone in LEC and LEA serum was assayed by RIA by Otsuka Assay Laboratories.

\section{RESULTS}

Expression of CAIII in LEC and LEA rats

CAIII levels varied significantly during development and aging of male rats as shown in Fig. 1 and Fig. 2. Expression of CAIII in LEA male rats increased rapidly from the age of 4 weeks to 24 weeks and after this period it gradually decreased. In LEA female rats, CAIII levels increased in a similar pattern to that of LEA males; however, the level was a third to a half of that of LEA males.

On the other hand, expression of CAIII in LEC male rats increased sharply from the age of 4 weeks and decreased with development of hepatitis. The expression of CAIII in LEC male rats during the immature period of 4 to 8 weeks was higher than that of LEA rats. LEC female rat liver expressed CAIII two times higher than LEA females, during the immature period, and then a sharp decrease was seen at the age of 12 weeks and it became constant.

\section{A. LEC male}

$\begin{array}{lllllllll}4 W & 8 W & 12 W & 16 w & 18 W & 42 W & 54 & \end{array}$

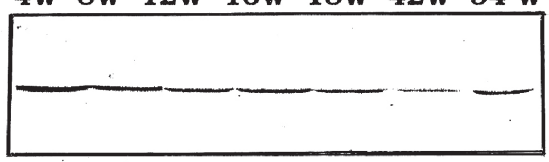

B. LEA male

$4 W \quad 8 W \quad 12 W \quad 16 W \quad 22 W \quad 24 W \quad 42 W 54 W$

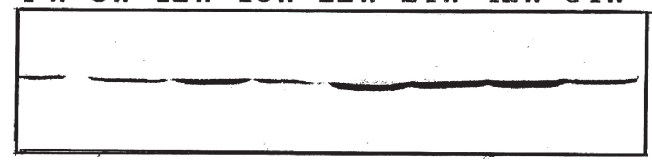

Fig. 1 Expression of carbonic anhydrase III in the liver of male LEA and LEC rats at various ages. Each sample $(40 \mu \mathrm{g}$ of cytosol fraction) was electrophoresed on 15\% SDS-polyacrylamide gel and analyzed by Western blotting, as described in "MATERIALS AND METHODS."

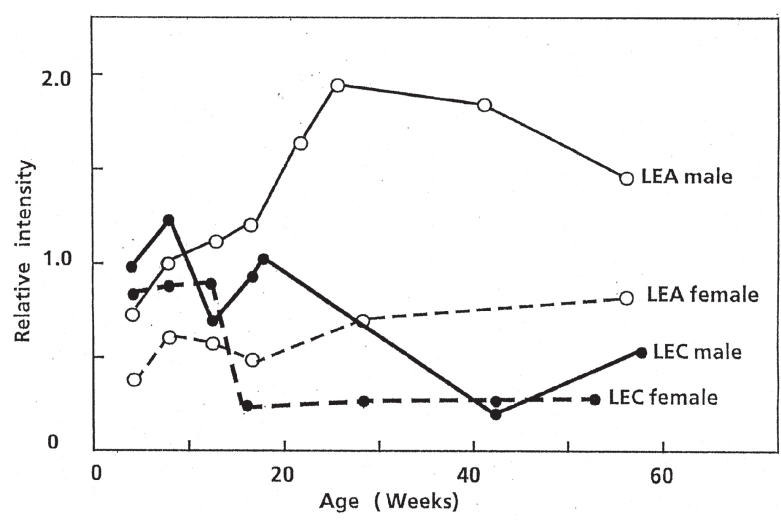

Fig. 2 A age-related changes in the contents of carbonic anhydrase III of liver cytosols in LEC and LEA rats. Each samples ( $40 \mathrm{\mu g}$ of protein of cytosol fraction) was electrophoreses on $15 \%$ SDS-polyacrylamide gel and analyzed by Western blotting, as described in "MATERIALS AND METHODS." The amount of proteins measured the density of a band by the densitometer. The amount of proteins in male LEC rats at 4 weeks was set to 1 , and the concentration of each protein was calculated as the relative value.

able but little is present in LEA rats of the same age (Fig. 3). The levels of testosterone in both LEC and LEA male rats increased during maturation, but there was no significant difference in testosterone level between LEC and LEA rats. From this result, it can be concluded that the level of testosterone may not be responsible for the expression of CAIII in LEC and LEA male rats. 


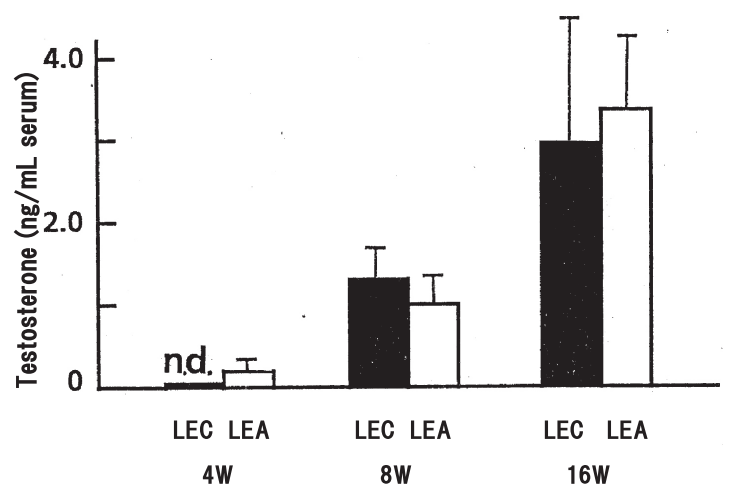

Fig. 3 Developmental changes in serum testosterone concentrations of male LEC and LEA rats. Each point represents the mean values obtained from the rats $(n=5)$. Standard deviations are shown using bars. The n.d. means "not detectable".

CAIII levels in hepatic hyperplastic nodules of LEC rats

Fig. 4 shows that surrounding tissue had higher level of CAIII than hyperplastic nodules and CAIII was almost absent in female LEC rat nodules. The levels of CAIII in the surrounding tissues of male rats were four times higher relative to female rats. The levels of CAIII in hyperplastic nodules of female rats were almost absent but present in males (about a half of that in surrounding tissue). From these results it is concluded that hyperplastic nodules may suppress the expression of CAIII.

\section{Immunohistochemical detection of CAIII}

Hyperplastic nodules were larger in size than hepatic lobules and compressed the surrounding hepatocytes. These hyperplastic nodules became much larger and developed into hepatomas. Hepatomas were of a well-differentiated type with a trabecular structure of tumorous hepatocytes containing round nuclei with large nucleoli. Surrounding hepatocytes were apparently normal. The results of immunohistochemical staining for CAIII as well as GST-P in hyperplastic nodular liver of LEC rats are shown in Fig. 5. GST-P foci could be clearly distinguished from surrounding hepatocytes on the basis of GST-P staining. CAIII was predominantly present in the surrounding hepatocytes, with little or none being detected in the hyperplastic nodules.

\section{DISCUSSION}

Age-related CAIII expressions in LEA and LEC In this study, we demonstrated that the expression pattern of CAIII in both LEA and LEC rat livers

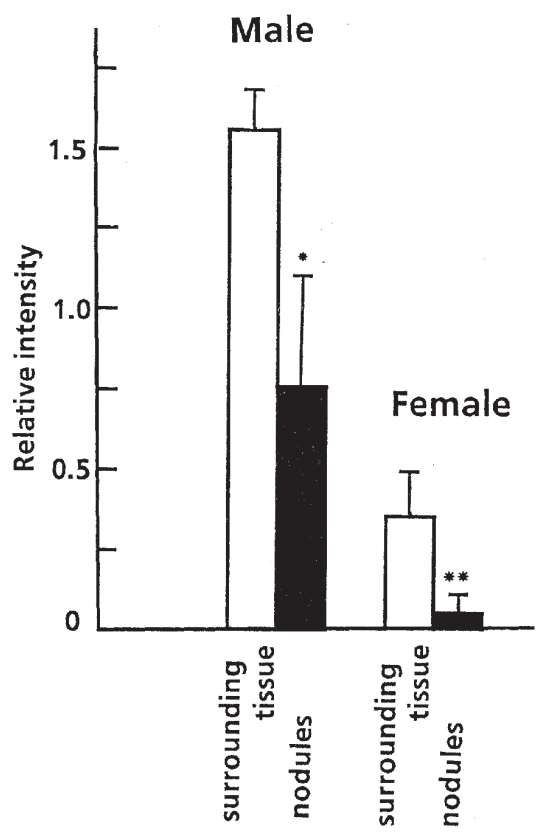

Fig. 4 Carbonic anhydrase III levels in hepatic hyperplastic nodules of male or female LEC rats determined by Western blot analysis. Hyperplastic nodules and surrounding tissues were excised carefully from the three livers of each male and female LEC rats at 52 weeks. ${ }^{*} P<0.05$, ${ }^{* *} P<0.025$.

was dimorphic, and CAIII was much more suppressed in LEC rats than in LEA rats of the corresponding sexes. Age-related expressions are shown in Fig. 2. Before puberty, CAIII levels of both sexes were higher in the LEC rats than corresponding LEA rats, but a rapid decrease was seen with the onset of hepatitis around 12 weeks. After recovery of hepatitis, CAIII levels declined in senescent LEC males. Therefore, it is possible that accelerated aging occurred in the LEC rats.

\section{Effect of testosterone on CAIII expression}

CAIII content in the liver is known to be affected by the levels of testosterone or estradiol (1). In neonatal male rats, testosterone is the predominant sex hormone, but it then declines in the prepubertal stage (up to day 30). The levels of testosterone increase at puberty and peaks at about day 70, after which fertility declines. It is known that castration of male rats leads to a marked reduction in liver CAIII concentrations that can be partially restored to control levels by testosterone replacement (1). Kasai et al. (13) examined the effects of sex hormones on fulminant hepatitis by administering exogenous sex hormones to ovarectomized or orchidectomized LEC rats. Estradiol did not cure the hepatitis but sup- 


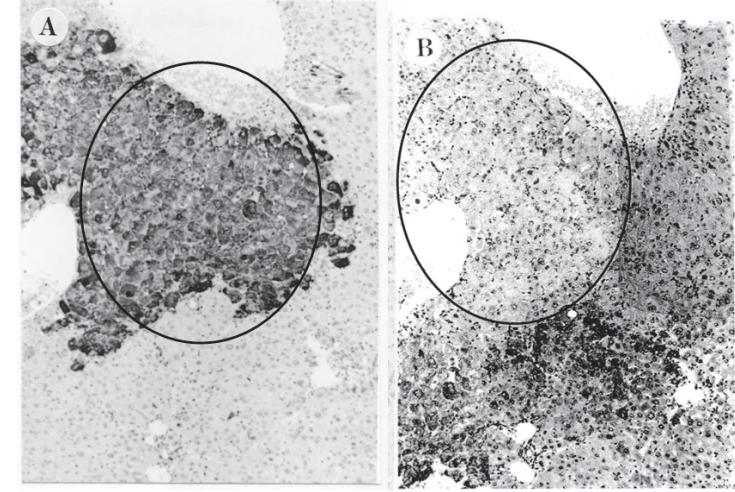

Fig. 5 Immunostaining of serial sections of LEC rat livers at 52 weeks. Serial thin sections were immunostained for CAIII and GST-P by the biotin-streptoavidin method. A, GST-P immunohistochemistry; hyperplastic nodule showing intense staining for GST-P. B, CAlll immunohistochemistry; surrounding normal hepatocytes showing low intensity staining of CAIII and the virtual absence of staining in hyperplastic nodules. $\times 250$.

pressed the deterioration and delayed the occurrence of fulminant hepatitis. In contrast, testosterone did not affect the onset of hepatitis but enabled the animal to survive the disease.

In the present study, the levels of testosterone in both LEC and LEA male rats increased during maturation, and there was no significant difference in testosterone level between LEC and LEA rats, as shown in Fig. 3. From these results, it can be concluded that the level of testosterone may be responsible for the expression of CAIII in LEA male rats. On the other hand, the expression patterns of CAIII in the LEC rats were different from those observed in the normal LEA rats.

The sexual dimorphism during hepatocarcinogenesis Sexual dimorphism has been extensively studied in LEC rats $(7,13,27)$. A sex difference in the growth of carcinoma has been observed in LEC rats, with the size of hepatocellular carcinomas being larger in males than in females (20), and preneoplastic foci appeared more frequently in livers of male LEC rats than those of females (34). On the other hand, cholangiofibrosis was more extensive in females than in males (20). The inducibilities of chromosomal aberrations and sister chromatid exchanges in bone marrow cells by cyclophosphamide were lower in females than in males of both strains, and there were strain differences between LEC rats and LEA rats of the same sex (the frequency was lower in LEC rats) (18).

We have investigated the expression of 14 forms of cytochrome P450 in the liver as well as changes in the testosterone hydroxylation activities of hepatic microsomes during the development of hepatitis in LEC rats (7). During the immature period at 4 to 8 weeks, the levels of the sex-specific forms of cytochrome P450 such as SYP2C11 and SYP3A2 were higher in LEC rats than in LEA rats, but in mature rats the levels of several sex-specific forms of P450 decreased markedly with aging or during development of hepatitis. These results suggest that the sexual dimorphism observed in cytochrome P450 expression may be involved in the inducibilities of hepatoma.

We compared to CAIII from the livers of 4-, 16and 52-week old LEC rats predisposed to hepatitis and hepatoma with that of LEA rats by two dimensional gel electrophoresis (21). In the present study, we confirmed that CAIII was dramatically greater in 4 week-old LEC rats than in 4 week-old LEA rats and that CAIII was lower in the LEC rats as compared with the LEA rats during aging, as shown in Figs. 1 and 2.

\section{Expression of enzyme markers during the hepatocar- cinogenesis}

Currently, several enzyme markers, such as ATPase, glucose-6-phosphatase, $\gamma$-GTP, and aldehyde dehydrogenase, are frequently used to identify preneoplastic and neoplastic lesions (27). We have previously shown that the Phase I enzymes in drug metabolism of the liver from LEC rats are markedly decreased, whereas those of Phase II are selectively increased $(30,31)$. It is also reported that LEC rats are particularly susceptible to hepatocarcinogens $(29,33,34)$.

It is well known that liver specific isozymesglucokinase and pyruvate kinase-are decreased during hepatocarcinogenesis. We showed that hexokinase may be used as an enzyme marker of the early stages of liver carcinogenesis (9). Hexokinase activity showed two phases; an increase at 4 weeks and a decrease at 10 weeks followed by an increase peaking at 20 weeks. These two phase patterns were shown for Mn-superoxide dismutase (32), $\gamma$-glutamyl transpeptidase and cytochrome P450 isozymes (10). These enzyme profiles in hepatocarcinoma showed a general tendency toward approaching the undifferentiated patterns in LEC rats. Fig. 5 shows that preneoplastic nodules could be clearly distinguished from surrounding hepatocytes as GST-P-positive and CAIII-negative. Therefore, CAIII is available for one of the negative markers such as glucose-6phosphatase and ATPase. 


\section{Effect of copper concentration on CAIII expression} The defective Atp7B protein in LEC rats is not able to incorporate cytosolic copper into the Golgi apparatus. This leads to the accumulation of copper in liver cytosol. Li et al. (17) reported that liver copper concentrations of 3- and 8-month-old LEC rats were over 50 and 40 times higher, respectively than those of age- and sex-matched LEA rats. Furthermore, Grimes et al. (4) demonstrated the decreased CAIII levels in the liver of the mouse mutant 'toxic milk' (tx) due to copper accumulation. In this study, expression of CAIII in both LEC male and female rats sharply decreased from the age of 18 weeks with development of hepatitis caused by copper accumulation as shown in Fig. 2.

In conclusion, it is most likely that the decrease in CAIII in the LEC mutant is a secondary consequence of copper accumulation, which developed into hereditary hepatitis and hepatoma with aging. Marked changes in liver CAIII content were also observed in developing and aging male rats. The expression patterns of CAIII in the LEC rats were different from those observed in the normal LEA rats. It will be of interest to examine further the molecular basis of the effect of copper on CAIII and to determine whether CAIII play any role in copper transport.

\section{Acknowledgements}

We are grateful to Dr. Tsutomu Kurosawa and Messrs Tomio Miura and Toshinori Ishikawa, the Institute of Experimental Animal Sciences of Osaka University Graduate School of Medicine, for maintaining LEC and LEA rats. We thank Dr. S. Shinn, Otsuka Assay Laboratories, Tokushima, Japan, for assaying testosterone in LEC rats. This work was supported in parts by Grants-in-Aid for Scientific Research and Grants-in Aid for Cancer Research from the Ministry of Education, Science, and Culture of Japan, and by a fund from Otsuka Pharmaceutical Co. Ltd.

\section{REFERENCES}

1. Carter ND, Shiels A, Jeffery S, Heath R, Wilson CA, Phillips IR and Shephard EA (1984) Hormonal control of carbonic anhydrase III. Ann N Y Acad Sci 429, 287-301.

2. Chegwidden WR (1991) Purification of the carbonic anhydrases, In: The Carbonic Anhydrases: Cellular Physiology and Molecular Genetics (Dodgson SJ, Tashian RE, Gros G and Carter ND, eds.), pp101-118, Plenum Press, New York.

3. Dodgson SJ (1991) The carbonic anhydrases: Overview of their importance in cellular physiology and in molecular genetics. In: The Carbonic Anhydrases: Cellular Physiology and
Molecular Genetics (Dodgson SJ, Tashian RE, Gros G and Carter ND, eds.), pp3-14, Plenum Press, New York.

4. Grimes A, Paynter J, Walker ID, Bhave M and Mercer JF (1997) Decreased carbonic anhydrase III levels in the liver of the mouse mutant 'toxic milk' (tx) due to copper accumulation. Biochem J 321, 341-346.

5. Hewett-Emmett D and Tashian RE (1991) Structure and evolutionary origins of the carbonic anhydrase multigene family. In: The Carbonic Anhydrases: Cellular Physiology and Molecular Genetics (Dodgson SJ, Tashian RE, Gros G and Carter ND, eds.), pp15-32, Plenum Press, New York.

6. Ikeda M, Ishii Y, Kato H, Akazawa D, Hatsumura M, Ishida T, Matsusue K, Yamada H and Oguri K (2000) Suppression of carbonic anhydrase III in rat liver by a dioxin-related toxic compound, coplanar polychlorinated biphenyl, 3,3',4,4',5pentachlorobiphenyl. Arch Biochem Biophys 380, 159-164.

7. Imaoka S, Sugiyama T, Taniguchi N and Funae Y (1993) Expression of cytochrome P450 in LEC rats during development of hereditary hepatitis and hepatoma. Carcinogenesis 14, 117-121.

8. Jabusch JR and Deutsch HF (1984) Sequencc of the highactivity equine erythrocyte carbonic anhydrase: N-terminal polymorphism (acetyl-serine/acetyl-Thr) and homologies to similar mammalian isozymes. Biochem Genet 22, 357-367.

9. Jain S, Jain SK, Sugiyama T and Taniguchi N (1991) High expression of hexokinase isozyme B during hepatocarcinogenesis in LEC rats. In: The LEC Rat: A New Model for Hepatitis and Liver Cancer (Mori M, Yoshida MC, Takeichi $\mathrm{N}$ and Taniguchi N, eds.), pp335-340, Springer-Verlag, Tokyo.

10. Jain SK, Suzuki K, Jain S, Sugiyama T and Taniguchi N (1991) Hypomethylation-associated expression of cytochrome P-450 and $\gamma$-glutamyl transpeptidase during hereditary hepatocarcinogenesis in LEC rats. In: The LEC Rat: A New Model for Hepatitis and Liver Cancer (Mori M, Yoshida MC, Takeichi N and Taniguchi N, eds.), pp162-168, Springer-Verlag, Tokyo.

11. Jeffery S, Carter ND, Clark RG and Robinson CAF (1990) The episodic secretory pattern of growth hormone regulates liver carbonic anhydrase III: Studies in normal and mutant growth-hormone-deficient dwarf rats. Biochem J 266, 69-74.

12. Jeffery S, Merry BJ, Holehan AM and Carter ND (1988) The effects of aging on carbonic anhydrase concentrations in rat liver and skeletal muscle. Biochem J 250, 303-305.

13. Kasai N, Kamimura E, Miyoshi I and Yoshida MC (1991) Reproductive performance and effects of pregnancy on the acute phase of hepatitis in LEC rats. In The LEC Rat: A New Model for Hepatitis and Liver Cancer (Mori M, Yoshida MC, Takeichi N, Taniguchi N, eds.) pp.11-19, Springer-Verlag, Tokyo.

14. Kim G, Lee, TH, Wynshaw-Boris A and Levine RL (2001) Nucleotide sequence and structure of the mouse carbonic anhydrase III gene. Gene 265, 37-44.

15. Laemmli UK (1970) Cleavage of structural proteins during assembly of the head of bacteriophage T4. Nature 227, 680 685.

16. Lehtonen J, Shen B, Vihinen M, Casini A, Scozzafava A, Supuran CT, Parkkila AK, Saarnio J, Kivela AJ, Waheed A, Sly WS and Parkkila S (2004) Characterization of CA XIII, a novel member of the carbonic anhydrase isozyme family. $J$ Biol Chem 279, 2719-2727.

17. Li Y, Togashi Y, Sato S, Enomoto T, Kang JH, Takeichi N, Kobayashi H, Kojima Y, Une Y and Uchino J (1991) Spontaneous hepatic copper accumulation in Long-Evans Cinnamon 
rats with hereditary hepatitis. A model of Wilson's disease. $J$ Clin Invest 87, 1858-1861.

18. Masuda R, Abe S, Yoshida MC, Sasaki M, Sugiyama T and Taniguchi N (1990) Cytochrome P-450 and chromosome damage by cyclophosphamide in LEC strain rats predisposed to hereditary hepatitis and liver cancer. Mutation Res 244, 309-316.

19. Masuda R, Yoshida MC, Sasaki M, Dempo K and Mori M (1988) Hereditary hepatitis of LEC rats is controlled by a single autosomal recessive gene. Lab Anim 22, 166-169.

20. Masuda R, Yoshida MC, Sasaki M, Dempo K and Mori M (1988) High susceptibility to hepatocellular carcinoma development in LEC rats with hereditary hepatitis. Jpn J Cancer Res 79, 828-835.

21. Nagase T, Sugiyama T, Kawata S, Tarui S, Deutsch HF and Taniguchi N (1991) Analyses of polypeptides in the liver of a novel mutant (LEC rats) to hereditary hepatitis and hepatoma by two-dimensional gel electrophoresis: Identification of P29/6.8 as carbonic anhydrase III and triosephosphate isomerase. J Comp Biochem Physiol 99B, 193-201.

22. Nishita T and Deutsch HF (1981) Isolation of equine muscle carbonic anhydrase in crystalline form. Biochem Biophys Res Commun 103, 573-580.

23. Petrukhin K, Fischer SG, Pirastu M, Tanzi RE, Chernov I, Devoto M, Brzustowicz ML, Cayanis E, Vitale E, Russo JJ, Matseoane D, Boukhgalter B, Wasco W, Figus AJ, Loudianos J, Cao A, Sternlieb I, Evgrafov O, Parano E, Pavone L, Warburton D, Ott J, Penchaszadeh GK, Scheinberg IH and Gilliam TC (1993) Mapping, cloning and genetic characterization of the region containing the Wilson disease gene. Nat Genet 5, 338-343.

24. Register AM, Koester MK and Noltmann EA (1978) Discovery of carbonic anhydrase in rabbit skeletal muscle and evidence for its identity with "basic muscle protein". J Biol Chem 253, 4143-4152.

25. Sasaki M, Yoshida MC, Kagami K, Takeichi N, Kobayashi H, Dempo K and Mori M (1985) Spontaneous hepatitis in an inbred strain of Long-Evans rats. Rat News Lett 14, 4-6.

26. Sato K (1988) Glutathione $S$-transferases and hepatocarcinogenesis. Jpn J Cancer Res 79, 556-572.

27. Sawaki M, Enomoto K, Takahashi H, Nakajima Y and Mori M (1990) Phenotype of preneoplastic and neoplastic liver lesions during spontaneous liver carcinogenesis of LEC rats. Carcinogenesis 11, 1857-1861.

28. Shiels A, Jeffery S, Phillips IR, Shephard EA, Wilson CA and Carter ND (1983) Sexual differentiation of rat liver carbonic anhydrase III. Biochem Biophys Acta 760, 335-342.
29. Sugiyama T, Matsunaga M, Jain SJ, Jain S, Ikeda Y and Taniguchi N (1991) Enhancing effect of a choline-deficient diet on alterations of hepatic drug-metabolizing enzymes in hepatitis- and hepatoma-predisposed rats (LEC rats). Jpn $J$ Cancer Res 82, 390-396.

30. Sugiyama T, Suzuki K, Ookawara T, Kurosawa T and Taniguchi N (1989) Selective expression and induction of cytochrome P-450PB and P-450MC during the development of hereditary hepatitis and hepatoma of LEC rats. Carcinogenesis 10, 2155-2159.

31. Sugiyama T, Takeichi N, Kobayashi H, Yoshida MC, Sasaki $\mathrm{M}$ and Taniguchi N (1988) Metabolic predisposition of a novel mutant (LEC rats) to hereditary hepatitis and hepatoma: alterations of the drug metabolizing enzymes. Carcinogenesis 9, 1569-1572.

32. Suzuki K, Nakata T, Seo HG, Miyazawa N, Sugiyama T and Taniguchi N (1991) Differential expression of $\mathrm{Mn}-$ and $\mathrm{Cu}$, $\mathrm{Zn}$-superoxide dismutases in various tissues of LEC rats. In: The LEC Rat: A New Model for Hepatitis and Liver Cancer (Mori M, Yoshida MC, Takeichi N and Taniguchi N, eds.) pp142-148, Springer-Verlag, Tokyo.

33. Suzuki K, Sugiyama T, Oogawara T, Kurosawa T and Taniguchi N (1991) High sensitivity to 5-azacytidine in LEC rats, a strain with a metabolic predisposition to hepatitis and hepatoma: Possible involvement of DNA methylation in the expression of cytochrome P-450 and $\gamma$-glutamyl transpeptidase. Biochem Int 23, 9-14.

34. Takahashi H, Enomoto K, Nakajima Y and Mori M (1990) High sensitivity of the LEC rat liver to the carcinogenic effect of diethylnitrosamine. Cancer Lett 51, 247-250.

35. Tanzi RE, Petrukhin K, Chernov I, Pellequer JL, Wasco W, Ross B, Romano DM, Parano E, Pavone L, Brzustowicz LM, Devoto M, Peppercorn J, Bush AI, Sternlieb I, Pirastu M, Gusella JF, Evgrafov O, Penchaszadeh GK, Honig B, Edelman IS, Soares MB, Scheinberg IH and Gilliam TC (1993) The Wilson disease gene is a copper transporting ATPase with homology to the Menkes disease gene. Nat Genet 5, 344350.

36. Xu Y, Campbell HA, Sattler GL, Hendrich S, Maronpot R, Sato K and Pitot HC (1990) Quantitative stereological analysis of the effects of age and sex on multistage hepatocarcinogenesis in the rat by use of four cytochemical markers. Cancer Res 50, 472-479.

37. Yoshida MC, Masuda R, Sasaki M, Takeichi N, Kobayashi H, Dempo K and Mori M (1987) New mutation causing hereditary hepatitis in the laboratory rat. $J$ Hered 78, 361-365. 